\title{
Construcción colectiva del conocimiento a través de la web social y motivación del alumnado: Proyecto de innovación docente aplicado al Grado en Comunicación
}

\author{
Laura TERUEL RODRÍGUEZ \\ Universidad de Málaga \\ Teruel@uma.es
}

\begin{abstract}
Resumen
Este artículo presenta los fundamentos de un proyecto de innovación docente aplicado en la docencia del Grado en Periodismo de la Universidad de Málaga y resultados. Atendiendo a la concepción pedagógica del EEES se ha planteado un proyecto educativo que parte de la coordinación entre materias, la implicación individual y grupal del alumnado en el aprendizaje y el desarrollo de competencias permanentes. La implementación de las herramientas digitales 2.0 para esta estrategia de aprendizaje se consideró fundamental.
\end{abstract}

Palabras clave: Innovación Educativa- Periodismo - competencias - motivación- aprendizaje colaborativo- web social

\section{Collective construction of knowledge through social web and student motivation: teaching innovation project applied to the Degree in Communication}

\begin{abstract}
This paper explains the theoretical fundaments of a teaching innovation project implemented in the Degree in Journalism from the University of Malaga, its development and results. Attending to the pedagogical conception of the EHEA, it has raised this educational project based on the coordination between subjects, individual and group involvement of students in learning and ongoing skill development. The implementation of digital tools 2.0 for this learning strategy has been considered essential.
\end{abstract}

Key words: Educational Innovation-Journalism - skills - motivation-social-web collaborative learning

\section{Referencia normalizada:}

Teruel Rodríguez, L. (2013) Construcción colectiva del conocimiento a través de la web social y motivación del alumnado: Proyecto de innovación docente aplicado al Grado en Comunicación. Historia y Comunicación Social. Vol. 18 No Especial Noviembre. Págs. 523-534.

Sumario: 1. Introducción. 2. Estado de la cuestión: El aprendizaje constructivo y colaborativo en el EEES. 2.1 La web social, herramienta fundamental para el aprendizaje 3. Planteamiento del proyecto docente: coordinación de materias e implicación del alumnado a través del uso de la web social. 4. Medición de los resultados didácticos: comparación de los resultados de Grado y Licenciatura. 5. Conclusiones. 6. Referencias Bibliográficas. 


\section{Introducción}

La implantación del Espacio Europeo de Educación Superior (EEES) ha conllevada una actualización de los parámetros por los que se guiaba la práctica docente universitaria. Si bien en los últimos años se ha venido fomentando el desarrollo de experiencias innovadoras en las universidades españolas, el planteamiento de Bolonia ha supuesto el empuje definitivo para acometer una reflexión sobre el proceso de aprendizaje en los niveles educativos superiores.

Este artículo presenta el planteamiento y los resultados del proyecto de un innovación docente aplicado en el Grado en Periodismo que parte de la concepción pedagógica EEES según la cual la enseñanza debe aspirar a implicar al alumnado para que se convierta en protagonista activo de su formación. Durante dos cursos se ha aplicado una metodología que ha requerido de la coordinación entre materias y que perseguía potenciar el desarrollo de competencias duraderas en el estudiante.

El principal objetivo de la propuesta es analizar cómo los estudiantes interiorizan los contenidos y desarrollan competencias de forma más duradera cuando se implican grupalmente en el aprendizaje y se introducen herramientas digitales adecuadas en el proceso formativo.

En este trabajo se describe, en primer lugar, el planteamiento teórico que subyace en esta experiencia, basado en el aprendizaje constructivo, la implicación grupal y la motivación individual. Posteriormente, se presentan los principales resultados de su aplicación que demuestran, en primer lugar, una valoración altamente positiva de los estudiantes de esta metodología; en segundo lugar, que las materias del Grado dejan de convertirse en contenidos estanco y el discente es capaz de aplicar los conocimientos adquiridos y las habilidades desarrolladas en todas las asignaturas. Ello prueba que la motivación se traduce en una implicación elevada del grupo en los espacios de construcción de contenido colaborativo - como wikis-. Por último, se comparan los resultados obtenidos en las materias comprendidas por este proyecto con otras que poseen programas similares pero que pertenece del plan antiguo -esto es, Licenciatura y no Grado-, lo que viene a refrendar los buenos resultados de esta experiencia.

La posibilidad de aplicar una metodología docente durante dos curso al mismo grupo ofrece una extraordinaria posibilidad de secuenciar el proyecto y evaluar los logros. Más aún, la comparación de los resultados obtenidos en Grado con los de Licenciatura, en materias teóricamente equivalentes, es interesante pues, por una parte, son estudiantes con prácticamente la misma edad y uso de entornos digitales y, por la otra, se demuestra cómo la práctica docente influye en el proceso de aprendizaje y en los resultados obtenidos; esto es, se evidencia cómo la implicación del profesor y el uso de herramientas adecuadas en el proceso formativo mejoran la formación. 


\section{Estado de la cuestión: EI aprendizaje constructivo y colaborativo en el EEES}

En las bases teóricas que inspiran el Espacio Europeo de Educación Superior se plasma que la participación de los estudiantes debe estar orientada hacia un aprendizaje activo, autorregulado, constructivo, situado y social. Existe, por tanto, un interés determinante en que el educando sea protagonista activo de su formación y reciba de los docentes y las instituciones académicas la posibilidad trazar su itinerario formativo. Así nos inscribimos en el marco del aprendizaje significativo y constructivo que persigue transferir las riendas del proceso de enseñanza del profesor al alumno (Beltrán Llera, 2003).

Lejos de las metodologías basadas en la repetición de contenidos, los acuerdos de Bolonia defienden que el alumno aprenda creando redes de conocimiento significativo. Esta estrategia le permitirá comprender y personalizar su proceso formativo de forma que la adquisición de conceptos y el desarrollo de habilidades sean permanentes.

Este nuevo paradigma docente ha implicado la potenciación tanto del trabajo autónomo y como del establecimiento de redes de trabajo colectivo -lo cual ha encontrado en internet una serie de herramientas idóneas-. La elección del itinerario personal del estudiante incrementa su motivación e implicación en el proceso de aprendizaje. Aumenta su responsabilidad en su formación pues determina su currículo académico a través de las materias interconectadas que le permitan alcanzar el perfil profesional que desea. En palabras de Bolarín, Moreno y Porto:

"Se demanda asimismo una implicación mayor del estudiante en este proceso, de forma que no sólo se preocupe de intentar asimilar todo aquello que se le transmite, sino que además sea capaz de mostrar cómo está reconstruyendo todos esos conocimientos para conformar su aprendizaje personal, de qué formas es capaz de elaborar su propia red de contenidos y puede utilizarla para enfrentarse a problemas de su campo disciplinar (en cualquiera de sus vertientes), con un mayor reconocimiento a las horas que debe dedicar a poder aprender autónomamente, al mismo tiempo que se incrementa la consideración de la necesidad de aprender a trabajar colaborativamente con otras personas (de igual o diferente especialización).” (2013: 440)

En este nuevo marco formativo, las metodologías docentes tradicionales no se antojaban suficientes para alcanzar estos objetivos. Se hacía necesario un mayor compromiso del docente y el discente así como una renovación de la concepción y las herramientas didácticas.

\subsection{La web social, herramienta fundamental para el aprendizaje}

Esta concepción docente encuentra en las nuevas tecnologías un aliado óptimo. Sin embargo, la simple utilización de estas herramientas en la práctica docente no es suficiente para considerar que mejoren cualitativamente la enseñanza. La transformación epistemológica debe estar propiciada por la creación de metodologías que favorezcan situaciones de aprendizajes colaborativos (Johnson y Johnson, 2009) y la autonomía del alumnado (Barberá y Rochera, 2008). Esto es, que los profesores implementen las 
potencialidades de internet $-\mathrm{y}$, en este caso, de la web social- a su proyecto docente para que no sea sólo una forma de replicar las prácticas tradicionales sino que se convierta en un entorno de aprendizaje enriquecido (Sclater, 2008).

Está generalizada la idea de que internet ya no es una biblioteca sino un lugar social (Iglesias, Lozano y Martínez, 2013) en el que cada estudiante tiene a su alcance herramientas para crear contenidos, intercambiar información, actualizarla, seleccionar aquello que nos interesa del flujo de datos constante y aplicar los contenidos teóricos a la praxis universitaria o profesional. La creación de comunidades virtuales para el aprendizaje (Cabero, 2006) ha proporcionado un espacio de colaboración que fomenta el aprendizaje colaborativo y el crecimiento personal de los alumnos en su trayectoria universitaria (Lapeña, Sauleda y Martínez, 2011).

Se ha hecho indispensable contar con internet y las herramientas digitales en la práctica docente de las Ciencias Sociales y, especialmente, en Periodismo. En primer lugar, porque su utilización sintoniza con las prácticas de consumo mediático y de ocio de los jóvenes. La adquisición de competencias se desarrollará con mayor naturalidad si se adapta a las inquietudes de los alumnos y les muestra que las aplicaciones que ya forman parte de sus vidas son, también, recursos para su formación. En segundo lugar, introducir la utilización de herramientas como sistemas de edición y transferencia de archivos en la nube o wikis, entre otros, en la docencia resulta conveniente en tanto dichas aplicaciones ofrecen soluciones fáciles, gratuitas y especializadas para el registro y tratamiento de datos, que son, a su vez, utilidades propias de los profesionales en gestión de contenidos mediáticos -nuevo perfil profesional de periodista según el Libro Blanco de la titulación (Sánchez y Teruel, 2012).

El significado de la web social o web 2.0 ha sido abordado en numerosas ocasiones de forma amplia (O'Reilly, 2006; Constantinides y Fountain, 2008; Ribes, 2009; Merlo- Vega, 2008). Las aproximaciones al concepto, lejos de ser cerradas, coinciden en que es un salto cualitativo en el desarrollo de internet porque favorece procesos de creación de contenidos colaborativos, posibilita la interrelación de diferentes bases de datos, el navegador web es la única herramienta necesaria para acceder a los servicios, implementa el uso del etiquetado como sistema para crear redes de conocimientos y posibilita la personalización total de los servicios y aplicaciones.

Las aplicaciones que ofrece la web social son útiles, por tanto, desde una concepción constructiva del aprendizaje, para el estudiante porque facilitan la adquisición de destrezas propias del profesional de la información -esto es, know how- como la habilidad para organizar de forma productiva la complejidad, para relacionar los conocimientos o el manejo eficiente de la lectura hipertextual (e-skills), entre otras.

La utilidad del presente trabajo reside en que muestra las mejoras en el aprendizaje, con resultados medibles, tras aplicar la coordinación de diferentes materias, potenciar la motivación del alumno e implementar nuevas herramientas digitales. Se propone, por tanto, un planteamiento de las nuevas posibilidades de la docencia constructiva y del aprendizaje colaborativo e individualizado partir de las posibilidades de la web social. 


\section{Planteamiento del proyecto docente: coordinación de materias e implicación del alumnado a través del uso de la web social}

En este apartado se describe la experiencia didáctica que se siguió en dos materias de Grado aplicada a un mismo grupo durante dos cursos consecutivos. Se trata de asignaturas muy diferentes tanto en el área de conocimiento al que pertenecen como en su situación en el plan de estudios: Investigación Básica Aplicada y Periodismo Político y Económico. Los objetivos educativos marcados para esta experiencia fueron:

Ofrecer una estrategia de aprendizaje significativo y permanente que permita al estudiante interiorizar contenidos y desarrollar competencias y habilidades de forma duradera.

Romper la concepción de 'conocimientos estanco' sobre las materias y fomentar la utilización de los conocimientos adquiridos en una asignatura en el resto de la etapa formativa y, por extensión, en su vida profesional -esto es, aprendizaje constructivo-.

Fomentar el aprendizaje grupal y el individual así como la motivación e implicación del estudiante en su proceso formativo.

Aplicar las herramientas que ofrece el entorno digital, especialmente la web social o 2.0, para la construcción del conocimiento colectivo. Descubrir al alumnado las utilidades académicas y profesionales de este entorno.

A continuación se explica la metodología desarrollada para alcanzar estos objetivos. No se ofrece una descripción detallada del planteamiento y los contenidos propios de cada asignatura sino que se presentan aquellas estrategias seguidas de forma coordinada en ambas materias específicamente para alcanzar las metas expuestas anteriormente.

La primera de las asignaturas fue impartida en el curso 2011/2012 por las profesoras Sánchez González y Teruel Rodríguez -turno de tarde y mañana respectivamente- ${ }^{-}$. Se trata de Investigación Básica Aplicada del segundo año del grado de Periodismo en la Facultad de Ciencias de la Comunicación de la Universidad de Málaga en la cual había matriculados 102 estudiantes. Al tratarse de una asignatura obligatoria y que para los alumnos es percibida como poco afín a la práctica periodística, se planificó una metodología didáctica que perseguía despertar su interés en la misma y motivarles individual y grupalmente a participar, en la que las herramientas de la web social serían determinantes.

1 El desarrollo de esta planteamiento docente está recogido en el capítulo: Sánchez González, M. y Teruel Rodríguez, L. (2013). "Herramientas colaborativas y aplicaciones 2.0 en la enseñanza de Métodos y Técnicas de Investigación en Comunicación. Investigar la Comunicación hoy". En Revisión de políticas científicas y aportaciones metodológicas: Simposio Internacional sobre Política Cientifica en Comunicación / coord. por Miguel Vicente Mariño Vol. 1, 2013 (COMUNICACIONES 1), ISBN 978-84-616-4124-6, págs. 87-102 
Para desarrollar las competencias y adquirir los conocimientos propios de la materia se proyectó una guía docente y un sistema de evaluación que comprendían tanto la interiorización de contenidos como la aplicación de las destrezas a un proyecto de investigación grupal. Crearon libremente equipos que trabajaron durante todo el semestre con el soporte de la tutoría de las docentes y con el aprendizaje teórico de las clases magistrales. Mediante la libre elección de tema de investigación y la voluntaria adscripción a los grupos conseguimos una mayor implicación del alumnado. La evaluación final contempló la valoración de estos trabajos colectivos pero, también, se realizó un ejercicio individual sobre la comprensión de conceptos.

Para el trabajo diario en clases encontramos en los recursos digitales aliados indispensables. La web social ofrece herramientas colaborativas que se convierten en un recurso sencillo que facilita la tarea docente y el aprendizaje. En una clasificación de los recursos de la web social que se aplicaron, podemos realizar una distinción entre:

Aquellos que permiten la autoría on line y colectiva y el seguimiento del desarrollo del proyecto (Peterson, 2009). La acumulación de conocimientos y sistematicidad, características propias del método científico, encuentran en estas herramientas espacios idóneos. El Campus Virtual de la UMA, basado en la plataforma Moodle, ofrece estas herramientas y es un recurso idóneo para el aprendizaje significativo.

El segundo tipo de recursos fueron los repositorios de contenidos o buscadores especializados como Dialnet, bases de datos especializadas en comunicación como Infoamérica o metabuscadores como Google Académico. Permiten al usuario un manejo constante, actualizado, gratuito y sin fronteras de material científico.

El apartado metodológico encontró en las herramientas de la web 2.0 un recurso vital. Se enseñaron diferentes instrumentos abiertos para elaboración colaborativa, registro y tratamiento de datos en la investigación de la comunicación. Así, por ejemplo, para el registro de datos cuantitativos o la realización de encuestas se trabajó con las opciones que ofrece Google Drive.

Del mismo modo, en último lugar, se explicaron diferentes aplicaciones que permiten presentar los resultados de las investigaciones en forma de flujo de datos o diagrama de secuencia que se adaptan a la investigación en web social.

Así pues, la utilización de estos recursos fue generalizada y continua durante las clases; ello generó una dinámica de aprendizaje colaborativo e implicación del alumnado. Los resultados cuantitativos de esta práctica se exponen en el siguiente apartado.

La segunda de las asignaturas integradas en este proyecto es la optativa Periodismo Político y Económico, impartida durante 2012/2013 en tercero por Laura Teruel Rodríguez. En la materia, situada dentro del área de conocimiento de Comunicación Especializada, se inscribieron 47 estudiantes. Al comienzo de las sesiones presenciales se planteó el sistema de evaluación que, junto con la realización de test de actualidad, incluía un examen final sobre el vocabulario técnico especializado explicado en clase y un trabajo grupo que debía versar sobre alguna de las temáticas propuestas por 
la docente. Para los objetivos que persigue este artículo nos centraremos en la aplicación de los conocimientos adquiridos el curso anterior, en primer lugar, y en el uso de las herramientas digitales colaborativas por parte del alumnado para su proceso de aprendizaje grupal e individual, posteriormente.

En este curso siguió potenciándose la libre elección de la línea de investigación y composición de los grupos en aras de fomentar la implicación individual y grupal. En vista de los buenos resultados obtenidos el año anterior en Investigación Básica Aplicada, los alumnos solicitaron a la docente que les recordara las normas que debe guiar un proyecto de investigación -basadas en la propuesta de Berganza y Ruiz San Román (2005)- y que dedicara una sesión a ello. Los estudiantes habían interiorizado la dinámica de los proyectos de investigación y quisieron extrapolar los conocimientos adquiridos a esta nueva prueba.

En lo referente al uso de la web social, ésta volvió a ser crucial para las clases. Su uso no fue propuesto por la docente sino que surgió, de forma espontáneo, por parte del grupo. Así, para el aprendizaje del vocabulario los alumnos decidieron crear un wiki en el que se repartieron los términos para su definición. La inmensa mayoría del grupo se inscribió voluntariamente en esta iniciativa de gestión colectiva del conocimiento. El wiki fue la herramienta colaborativa usada a gran escala pero hubo un interesante catálogo de recursos empleados para el trabajo grupal que, del mismo modo, fueron escogidos voluntariamente por ellos. En la encuesta anónima realizada al concluir el curso a través del campus virtual, se les preguntó por las facilidades que encontraron en esta y otras herramientas de la web social para su trabajo en este curso. Sus resultados se ofrecen en el siguiente epígrafe.

Al igual que en el curso anterior, el grupo optó por usar herramientas de la web 2.0 primero para la gestión colectiva del conocimiento; segundo, para la localización de fuentes teóricas de referencia y consulta hemerográfica; tercero, en el apartado metodológico para la obtención de datos; y, por último, para la presentación de los resultados.

\section{Medición de los resultados didácticos: comparación de los resultados de Grado y Licenciatura}

En este apartado se exponen los indicadores cuantitativos y cualitativos que permiten medir el éxito de esta experiencia docente y se comparan los resultados obtenidos con los del último curso de la Licenciatura de Periodismo en las materias más similares a las de Grado. El valor añadido de esta comparación reside en poder analizar si hay una mejora observable en los resultados académicos de materias homologables al aplicar metodologías diferentes.

Con respecto a las calificaciones y sistemas de evaluación, en Investigación Básica Aplicada los resultados cuantitativos fueron satisfactorios: el curso fue superado con una nota media de 7,17 en la convocatoria de junio y un $72 \%$ de estudiantes con la 
evaluación continua. En Periodismo Político y Económico las cifras fueron incluso mejores: el $80.8 \%$ de los alumnos se acogió al sistema de evaluación continua y, entre los evaluados en junio, la media fue de 7,3.

Para estudiar el uso de las herramientas de la web social que voluntariamente utilizaron los alumnos se hizo una encuesta una vez impartida la segunda de las materias -en tanto en la primera de ellas la web social fue introducida por las docentes-. Destacó el éxito del wiki: la nota media específica del test de vocabulario, para el cual los estudiantes se basaron en el contenido creado colectivamente a través de esta aplicación, fue de 8.15. En la encuesta realizada anónimamente al grupo, puntuaron con un valor de 4.3 sobre 5 su consulta y contribución al wiki. Esto es una muestra de la utilidad de este instrumento para el trabajo colectivo y continuo; la buena nota evidencia que los estudiantes se responsabilizaron a la hora de realizar las definiciones de los términos de forma comprensible para los compañeros y de corregirlos cuando lo entendían mejor tras la explicación en clase.

Junto a esta herramienta, destacó la utilización de Facebook con fines académicos y para trabajar en equipo (71\%). Frente a ésta, otra red social como Twitter -mediante la cual se recomendaban noticias interesantes para la asignatura a través de un usuario creado para ello- fue valorada positivamente por el $58 \%$. Dentro de las utilidades que ofrece internet para la organización del trabajo, el $86 \%$ de los alumnos manifestó haber utilizado herramientas gratuitas colaborativas como Google Docs para gestionar los apuntes de clase.

En segundo lugar, en el apartado de los recursos de investigación, el 71\% de alumnos consideró imprescindible el uso de buscadores especializados como Dialnet o Google Académico. El 86\% afirmó haber descubierto estas herramientas digitales en Investigación Básica Aplicada. Ello corrobora que aún tratándose de una generación digital, estas utilidades no figuraban en su mapa de uso de internet pero que, una vez explicadas durante el Grado, las interiorizaron y entendieron su utilidad para su formación académica. Por ello, preguntados por los conocimientos adquiridos y las destrezas desarrolladas el curso anterior el $82 \%$ admitió que le había sido útil y lo había aplicado a varias asignaturas. Supieron ponerlos en prácticas en materias diferentes y, con ello, enriquecieron su aprendizaje y rompieron los compartimientos estancos en que a veces quedan los conocimientos adquiridos en otros cursos.

Las respuestas cualitativas a esta encuesta sobre la gestión colectiva del conocimiento, la implicación individual y grupal del alumnado facilitado a través del uso de la web social ofrecieron una amplia gama de contestaciones que pueden organizarse en torno a cuatro aspectos:

Estas herramientas permiten trabajar simultáneamente, a tiempo real. Se aprovechan las sinergias positivas de trabajar a pesar de la lejanía. Así lo explicaba un estudiante: "Que todos podamos ver lo mismo en nuestra pantalla y trabajar sobre ello a tiempo real. Esto, al final, acaba dando resultados porque se agiliza la tarea en la mayoría de casos". 
Son aplicaciones que permiten organizarse cuando no hay tiempo para quedar. Por tanto, de forma complementaria a la primera respuesta, no sólo facilitan trabajar a la vez sobre un texto sino, también, de forma secuenciada: "La ventaja fundamental es poder elaborar el trabajo sin tener que quedar de forma física y facilidad en la comunicación entre los componentes del trabajo" / "Sobre todo la inmediatez con la que se trabaja, es decir la rapidez de compartir documentos con compañeros de tu grupo a una velocidad óptima para finalizar el trabajo a tiempo".

En tercer lugar, destacaban que la web social permite mejorar la comunicación del equipo, hacer las discusiones más profundas -basadas en documentos y datos-, un mejor reparto de las tareas y que el proyecto final se beneficie de la acumulación de conocimientos y correcciones de todos: "Relaciones de interacción, o sea, se intercambian conocimientos, lees formas de ver las cosas distintas a la tuya, y eso es enriquecedor para cualquier persona, y en concreto en los trabajos ayuda que expertos en la materia se animen a explicar aspectos de su ámbito de especialización".

Por último, reconocían las ventajas de los buscadores digitales de contenidos científicos: "Incorporar fuentes de información diversas" / "Amplia el número de documentos que pueden ser usados para realizar el trabajo".

Es interesante reseñar que los alumnos concebían estas herramientas como una ayuda organizativa pero que valoraban especialmente la interacción cara a cara: muchos apostillaron que quedar en persona es más enriquecedor.

Los datos cuantitativos permiten realizar una comparación de los resultados con materias similares de Licenciatura: esto es, con las obligatorias de cuarto curso Métodos y Técnicas de Investigación en Comunicación en el caso de Investigación Básica Aplicada y de Comunicación Especializada con Periodismo Político y Económico. Se trata de asignaturas similares en su temario y que fueron impartida por la misma profesora pero en las que ni el uso de la web social ni la metodología de implicación docente eran homologables, por más que se persiguiera motivar al alumnado y ofrecer unos contenidos actualizados y atrayentes con la misma intensidad. En el caso de Métodos y Técnicas, la nota media de los evaluados en junio en el curso 2011/2012 fue de 6.08 (83 alumnos). En Comunicación Especializada, en el último curso de la Licenciatura (2012/2013), la prueba teórica alcanzó una media de 5.9 entre los 91 estudiantes examinados en junio. La diferencia de más de un punto de media en ambas materias es elocuente.

\section{Conclusiones}

El propósito de este artículo es mostrar cómo se logra un aprendizaje constructivo y duradero implementando este proyecto de innovación docente y las herramientas que ofrece la web social en las Ciencias Sociales. Se trata de una experiencia desarrollada en dos asignaturas del grado de Periodismo en la UMA y, por ello, sus resultados tienen un alcance moderado. Pero no ofrece dudas sobre la veracidad de las 
hipótesis de las que partía para romper el aprendizaje repetitivo, lograr la motivación e implicación grupal del alumno y, paralelamente, mantener su espacio individual de aprendizaje y, por último, para enseñarles a sacarle partido académico a las herramientas digitales.

A modo de conclusiones finales, los resultados hallados demostraron que:

a) Frente a la idea de nativos e inmigrantes digitales de Prensky (2001) -que asociaba las habilidades digitales a un factor generacional-, White (2011) ha acuñado los conceptos de visitantes frente a residentes digitales para diferenciar comportamientos en función no de la edad sino de la actitud de cada individuo ante las nuevas tecnologías Así pues, si bien es cierto que la mayoría de jóvenes están habituados al manejo de tecnologías y de la web social, ello no se traduce automáticamente en el dominio de las competencias adecuadas para un uso desde la perspectiva académica. El elevado porcentaje de estudiantes (86\%) que descubrió las herramientas didácticas de la web 2.0 a través de las asignaturas integradas en este proyecto es muestra de ello. Más aún, el hecho de que los estudiantes de Licenciatura desconociera estas herramientas refrenda, sin duda, que no es un factor solo generacional: es imprescindible introducir las mismas para el proceso formativo para que los jóvenes sean residentes digitales.

b) Las calificaciones -más de un siete en ambos cursos-, tomadas como referente cuantitativo, avalan el éxito del proyecto. En este caso, una materia técnica y otra del área de Comunicación Especializada mantienen una línea de continuidad metodológica que afianza los conocimientos y habilidades adquiridos por los alumnos. La comparación con los resultados en las materias homologables en Licenciatura -con notas medias más de un punto inferiores- que no participaron de esta experiencia refrenda estos datos.

c) Los estudiantes interiorizaron y aplicaron voluntariamente las técnicas de trabajo individual y grupal porque constataron que mejoraban sus resultados académicos y les facilitaba la tarea. En el segundo curso la utilización de estas herramientas fue optativa y un $82 \%$ las empleó. Ello supone una alta implicación del estudiante en el entorno colectivo de aprendizaje y consigue la motivación al observar que su trabajo constante, favorecido por las herramientas digitales y reconocido a través de la evaluación continua, le beneficia académicamente.

d) La web social fue una herramienta indispensable para alcanzar estos resultados. No se replicó la práctica docente tradicional con estas utilidades sino que se desarrolló una iniciativa innovadora a partir de la implementación de la web 2.0 y que la transformó radicalmente. Por ello, cabe concluir que las aplicaciones de la web social coadyuvan a potenciar el desarrollo de e-skills o habilidades permanentes y adecuadas al entorno cambiante en que se desarrolla actualmente la labor del profesional de la información. 


\section{Referencias Bibliográficas}

\section{Libros}

BERGANZA CONDE, M.R. y RUIZ SAN ROMÁN, J. A. (2005). Investigar en comunicación. Editorial McGraw-hill, Madrid.

DÍAZ BARRIGA Arceo, F., \& HERNÁNDEZ ROJAS, G. (2002). Estrategias docentes para un aprendizaje significativo. Una interpretación constructivista. México, DF: McGraw-Hill.

RUÉ, J. (2007). Enseñar en la Universidad: El EEES como reto para la Educación Superior. Narcea Ediciones.

Capítulos o artículos en libros o revistas en papel

BARBERÁ, E. \& ROCHERA, M. J. (2008). "Los entornos virtuales de aprendizaje basados en el diseño de materiales autosuficientes y el aprendizaje autodirigido". En Coll, C., \& Monereo, C. (Eds.). Psicología de la educación virtual, (pp. 179-193). España: Morata.

BELTRÁN LLERA, J. (2003) "Estrategias de aprendizaje" En Revista de Educación, Universidad Complutense de Madrid. núm 332. Pp55-73

BOLARÍN MARTÍNEZ, M.J.; MORENO YUS, M.A.; PORTO CURRÁS, M. (2013): "Coordinación docente e interdisciplinariedad: análisis de su contribución a la adquisición de competencias docentes y discentes". En Revista de Docencia Universitaria. REDU. Volumen 11 (2), pp. 443-462

CABERO, J. (2006). "Comunidades virtuales para el aprendizaje. Su utilización en la Enseñanza" En Edutec. Revista Electrónica de Tecnología Educativa, 20, Enero 06

CONSTANTINIDES, E., \& FOUNTAIN, S. J. (2008). "Web 2.0: Conceptual foundations and marketing issues" En Journal of Direct, Data and Digital Marketing Practice,9(3), 231-244.

GEWERC BARUJEL, A. (2005). "El uso de weblogs en la docencia universitaria". En RELATEC: Revista Latinoamericana de Tecnología Educativa, 4(1), 9-24.

GONZÁLEZ, M. R. (2008). "El enfoque por competencias en el EEES y sus implicaciones en la enseñanza y el aprendizaje". En Tendencias pedagógicas, 13, 79-105.

IGLESIAS MARTÍNEZ, M.J.; LOZANO CABEZAS, I.; MARTÍNEZ RUIZ, M.A. (2013). "La utilización de herramientas digitales en el desarrollo del aprendizaje colaborativo: análisis de una experiencia en Educación Superior" En Revista de Docencia Universitaria. REDU. Vol.11 (2) Mayo-Agosto. pp. 333-351 Disponible en http://red-u.net/redu/index.php/REDU

JOHNSON, D. W. y JOHNSON, R. T. (2009). “An educational psychology success story: interdependence theory and cooperative learning". En Educational Researcher, 38 (5), 365-379

LAPEÑA, C., SAULEDA, N. y MARTÍNEZ, M. A. (2011). "Los programas institucionales de acción tutorial: una experiencia desarrollada en la Universidad de Alicante” En Revista de Investigación Educativa, 29 (2), 341-361. 
RIBES, X (2007) "La Web 2.0. El valor de los metadatos y de la inteligencia colectiva" En Telos: Cuadernos de comunicación e innovación. N ${ }^{\circ}$ 73, 2007. Págs. 36-43

SÁNCHEZ GONZÁLEZ, M. y TERUEL RODRÍGUEZ, L. (2013). "Herramientas colaborativas y aplicaciones 2.0 en la enseñanza de Métodos y Técnicas de Investigación en Comunicación. Investigar la Comunicación hoy". En Revisión de políticas científicas y aportaciones metodológicas: Simposio Internacional sobre Política Científica en Comunicación / coord. por Miguel Vicente Mariño Vol. 1, 2013 (COMUNICACIONES 1), ISBN 978-84-616-4124-6, págs. 87-102

Artículos en publicaciones web

MERLO- VEGA, J. A. "Las diez claves de la Web social". En Anuario ThinkEPI 2009, EPI SCP, pp. 34-36. Capturado el 20 de agosto de 2013 en: http://dialnet. unirioja.es/servlet/dcfichero_articulo?codigo $=3012969$

O’REILLY, T. (2006). "Qué es Web 2.0. Patrones del diseño y modelos del negocio para la siguiente generación del software" En Boletín de la Sociedad de la Información: Tecnología e Innovación. Capturado el 3 de agosto de 2013: http:// sociedadinformacion.fundacion.telefonica.com/DYC/SHI/seccion=1188\&idio$\mathrm{ma}=\mathrm{es}$ ES\&id $=2009100116300061 \&$ activo $=4$. do? elem $=2146$

PRENSKY, M. (2001) “Digital Natives, Digital Immigrants Part 1", En On the Horizon, Vol. 9 Iss: 5, pp.1 - 6. Capturado el 5 de septiembre de 2013 en: http://www. marcprensky.com/writing/Prensky\%20-\%20Digital\%20Natives,\%20Digital\%20 Immigrants\%20-\%20Part1.pdf

SCLATER, N. (2008). Web 2.0, Personal Learning Environments, and the Future of Learning Management Systems. Research Bulletin, 13 (2008), 13pp. Recuperado de http://educase.edu/ecar el 10 de septiembre de 2013

WHITE, David S. (2011). "Visitors and Residents: A new typology for online engagement". En First Monday; Vol. 16, num. 9, Capturado el 12 de septiembre de 2013 en: http://firstmonday.org/htbin/cgiwrap/bin/ojs/index.php/fm/article/view/3171

\section{La autora}

Laura Teruel. Profesora del Departamento de Periodismo de la Facultad de Ciencias de la Comunicación de la Universidad de Málaga y miembro del grupo de investigación SEJ- PAIDI 391 "Nuevos Espacios de Comunicación "de la Junta de Andalucía desde 2005 y del proyecto "Nuevos Escenarios en la investigación aplicada al estudio del sistema de medios" Plan Nacional I+D MEC SEJ 2006-14561/ soci. Doctora en Periodismo por la UMA, Máster en Política y Democracia por la Facultad de Ciencias Políticas y Sociología de la UNED, y Especialista Universitaria en Diseño y creación de materiales web para una asignatura en internet por la UMA. Especializada en Periodismo Político, directora del I y II Curso de Introducción al Periodismo Político- título propio de la UMA-, y en estudios de género. 